\title{
A Central Nervous System Focused Treatment Program for People with Frozen Shoulder: A Feasibility Study.
}

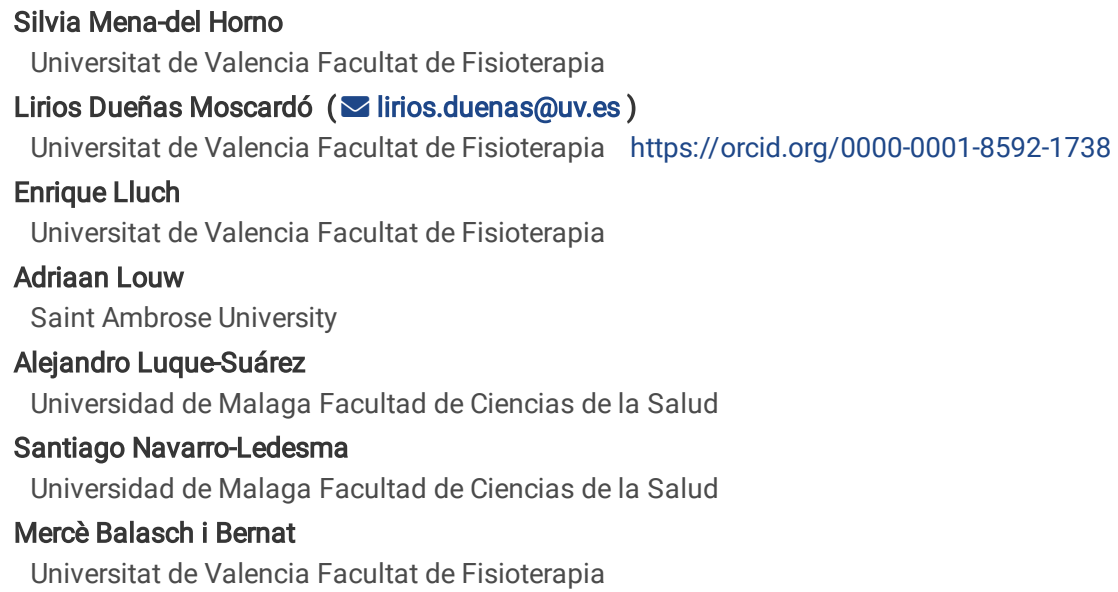




\section{Abstract}

Background: Frozen shoulder (FS) is a highly disabling pathology of poorly understood etiology, which is characterized by the presence of intense pain and progressive loss of range of motion (ROM). The aim of this study was to evaluate the feasibility and clinical impact of a CNS-focused treatment program for people with frozen shoulder (FS).

Methods: 10 subjects with primary FS received a 10 weeks CNS-focused intervention including sensory discrimination training and graded motor imagery techniques delivered as clinic sessions (60 minutes) and home therapy (30 minutes five times per week). Measurements were taken at baseline, after a 2weeks washout period, after treatment and at three months follow-up. The Shoulder Pain and Disability Index (SPADI) was the primary outcome. Secondary measures were: feasibility-related outcomes, self-reported shoulder pain, active and passive range of motion, two-point discrimination threshold (TPDT), left/right judgement task (LRJT), fear-avoidance (Tampa Scale for Kinesiophobia), pain catastrophization (Pain Catastrophizing Scale) and pain sensitization (Central Sensitization Inventory).

Results: $70 \%$ of participants completed the treatment. No significant changes were found after washout period except for TPDT ( $p=0.02$ ) and SPADI $(p=0.025)$. Improvements in self-reported shoulder pain $(p=0.028)$ and active shoulder flexion $(p=0.016)$ were shown after treatment $(p=0.028)$ and follow-up $(p=0.001)$ and in SPADI at follow-up $(p=0.008)$. No significant changes were observed in TPDT, LRJT, fear-avoidance, pain catastrophization and pain sensitization.

Conclusions: a CNS-focused treatment program might be a suitable approach to improve pain and disability in FS but further research is needed to draw firm conclusions.

Trial registration: Clinical Trials. NCT03320200. Registered 25 October 25 2017, https://clinicaltrials.gov/ct2/show/NCT03320200? term $=$ NCT03320200\&draw $=2 \&$ rank $=1$

\section{Introduction}

Frozen shoulder (FS) is a highly disabling pathology of poorly understood etiology [1], which is characterized by the presence of intense pain and progressive loss of range of motion (ROM) [2]. FS is present in $2 \%-5 \%$ of the general population, specially in women aged between 40 and 65 years and its exact etiology is currently unknown [3].

Many treatments have been proposed for FS including conservative (i.e. manual therapy) [4], and non-conservative approaches (i.e. arthroscopic capsular release) [5]. However, none of these interventions influence the natural history of this condition so innovative research seems necessary [6]. Some autors have suggested an involvement of central pain mechanisms secondary to continuous nociception characteristic of the early stages of FS [6]. Two systematic reviews showed preliminary evidence that central pain mechanisms may contribute to shoulder pain of different etiologies [7, 8], but other studies questioned those findings [9]. Importantly, these reviews did not included people with FS, so the role of the central nervous system (CNS) in FS remains speculative.

Different approaches targeting the CNS (e.g. graded motor imagery (GMI), tactile discrimination training) have been applied in a variety of chronic musculoskeletal pain disorders with promising results [10,11]. Specific to shoulder pain, Louw et al [12] presented a case-series where a CNS-focused treatment program based on a brief mirror therapy intervention was applied in subjects with shoulder pain and limited active ROM. This approach showed statistically significant improvements in pain, pain catastrophization, fear-avoidance and shoulder flexion active ROM [12]. However, only 8.7\% of the sample presented a diagnosis of FS. Similarly, Sawyer et al (2018) applied a combination of pain neuroscience education, tactile discrimination training and GMI in an individual with FS. The patient reported significant improvements in pain, fear of movement and active ROM [13]. Further high-quality research about the effectiveness of CNS-focused treatments in people with FS is needed.

The aim of this study was to evaluate the feasibility and clinical impact when implementing a CNS-focused treatment program for people with FS. The results of this study will inform of the appropriateness to conduct a randomized controlled trial on this topic.

\section{Materials And Methods Sample recruitment}

A convenience sample of 10 subjects diagnosed with FS was recruited. Since there is no gold standard to diagnose FS, diagnosis was stablished by a physician based on clinical examination, exclusion of other pathologies and imaging [14]. Patients included should present with primary or idiopathic FS, a limitation in passive external rotation $>50 \%$ compared to the unaffected shoulder or less than $30^{\circ}$ of passive external rotation, and a ROM loss $>25 \%$ in at least two movement planes [15]. Additionally, pain and movement restriction should be present for at least one month having either reached a plateau or worsened [15] and radiographs had to be normal (with the exception of osteopenia of the humeral head and calcific tendinosis) [16].

Patients that presented with locked dislocations, arthritis, fractures or avascular necrosis were excluded. Furthermore, those subjects not understanding Spanish language, having previous upper quadrant region surgery during the last year, any skin or medical condition preventing them from receiving tactile stimuli on the shoulder, any neurological or motor disorder, visually impaired or having a diagnosed psychopathology were excluded from the study.

\section{Procedures}


This feasibility study involved a 10-week CNS-focused intervention and periodic assessment of participants. All outcome measurements were performed at baseline (T-1) and after a 2 week period of "washout" with no intervention (T0) [17]. After this initial assessment participants began the treatment and were again measured at the end of treatment (3 months after baseline; T3) and at three months follow-up (T6) (Fig. 1).

The CNS-focused intervention consisted of a 10-week treatment program (1 session/week) delivered as 60 min sessions. In addition, participants performed a 30-minutes home training program five times per week during those 10 weeks. The CNS-focused intervention included discussion of the participant's shoulder pain experience from a pain neuroscience perspective provided in session 1 plus graded sensory discrimination training and GMI [18]. The physiotherapist performing treatment (SM) had a post-graduate degree in manual therapy and was trained in how to perform the treatment by another researcher (ELL) with 10 years working experience in the use of these interventions.

\section{Primary outcome measure}

The primary outcome measured was self-reported shoulder pain and disability measured with the Shoulder Pain and Disability Index (SPADI) [19]. The SPADI is a 13-items shoulder function index assessing pain and disability related to shoulder dysfunction [20]. Each item is scored by a numeric scale (0-10) and the total score ranges from 0 to 100 points. A higher score indicates greater disability. The Spanish version of the SPADI has shown high internal consistency and excellent test-retest reliability [21]. The Minimal Clinically Important Difference (MCID) for the SPADI ranges from 8 to 13 points [22].

\section{Secondary outcome measures}

Different feasibility outcomes were considered as secondary: timely recruitment, number of participants completing treatment, treatment compliance and barriers (with clinic and home training sessions), and number of patients measured at follow-up. To assess treatment adherence patients were provided with a diary to record their compliance with therapy [23]. In addition, patients were asked whether any difficulties with treatment compliance had appeared from one session to another.

Additionally, other secondary outcome measures were collected: self-perceived shoulder pain, active and passive ROM, and changes in tactile acuity and laterality judgement performance, Tampa Scale for Kinesiophobia (TSK-11), Central Sensitization Inventory (CSI) and Pain Catastrophizing Scale (PCS).

\section{Self-perceived shoulder pain}

Participants' self-perceived shoulder pain was evaluated with the Numeric Pain Rating Scale (NPRS) anchored with 0 ("no pain") and 10 ("pain as bad as you can imagine"). Patients reported their most intense pain over the last week, least intense pain over the last week, average pain intensity over the last week, and pain at that moment. The scores were averaged to calculate a final pain intensity score [24]. NPRS is a valid and reliable measure in patients with shoulder pain [25]. The minimal detectable change (MDC) of the NRPS for patients with shoulder pain is 2.5 points and the MCID is 1.1 points [25].

\section{Shoulder range of motion (ROM)}

Shoulder flexion and active and passive external rotation at $0^{0}$ of abduction of the affected shoulder were measured with a goniometer with the patient seated. To allow consistency of pre and post-therapy measurements, skin marks were placed for goniometric measurements. There is good reliability and validity of goniometric shoulder ROM measurements [26]. The MDC for shoulder flexion, abduction and external rotation ranges from $11^{\circ}$ to $16^{\circ}$ [27].

\section{Tactile acuity}

Tactile acuity was assessed with the two-point discrimination threshold (TPDT). A mechanical sliding calliper with a 1-mm precision (Duratech TA-2081) was used to calculate the TPDT. Participants were positioned in sitting and a point $5 \mathrm{~cm}$ distal to the lateral border of the acromion was marked on the painful shoulder. In order to standardize the testing region, this point was always kept between the two calliper points and measurements were performed in the longitudinal direction of the arm [28]. An ascending and a descending run of measurements were completed. The calliper distance was first gradually increased from $0 \mathrm{~mm}$ in $5 \mathrm{~mm}$ steps until the participant perceived two points instead of one. The descending run began with the calliper points separated $30 \mathrm{~mm}$ more than the TPDT value obtained from the ascending run, followed by decrements of $5 \mathrm{~mm}$. A mean TPDT value was obtained from the two threshold scores and used for analysis.

\section{Laterality judgement}

Laterality judgement was assessed with a left/right judgement task (LRJT) using the NOI ${ }^{\text {Tw }}$ online program. A total of 30 shoulder pictures (context mode) were presented to participants on a laptop in a random order and they were instructed to decide as quickly as possible but without guessing whether the picture showed the right or left shoulder thus making a response. Accuracy and mean response time were recorded. The LRJT was performed twice. The first block of images was used for task familiarization and data from the second block was used for analysis [29]. The normative mean (SD) response time and mean (SD) accuracy of this LRJT is 1738(741) ms and 93.5(9.2)\%, respectively [30].

\section{Questionnaires}

Fear avoidance was assessed with the Spanish version of the TSK-11 [31]. The TSK-11 is an 11-item questionnaire used to assess fear of movement or (re)injury during movement [32]. The total score ranges from 11 to 44 with higher scores indicating more fear-avoidance behavior. The TSK-11 has shown acceptable internal consistency and validity in both subjects with acute and chronic musculoskeletal pain [31]. The MDC for the TSK-11 is 5.6 [33]. The Spanish version of the CSI was used to assess different symptom dimensions related to central sensitization [34]. The CSI has high test-retest reliability and 
internal consistency [34]. Pain catastrophization was assessed with the Pain Catastrophizing Scale (PCS). PCS consists of 13 items and the total score ranges from 0 to 52 [35]. A total PCS score of 30 represents a clinically relevant level of catastrophizing [35].

\section{CNS-focused treatment program}

Prior to starting treatment, participants were given an explanation of the study. Patients were shown a picture of the 'brain map' (homunculus) and taught how, when people are in pain, the map becomes "less sharp" since it's not being moved and it is believed that when the map is sharpened, it may help reduce their pain even movements [12]. By using sensory discrimination training and GMI, the therapy aimed to sharpen the brain shoulder map and thus improve pain and movement. The CNS-focused treatment included graded sensory discrimination training and GMI training techniques. A full description of the treatment can be found elsewhere [36].

\section{Statistical analysis}

Statistical analysis was conducted using IBM SPSS Statistics 21 . Normality of the data was assessed using the Shapiro-Wilk test. Study findings are expressed as the mean and standard deviation or $95 \%$ confidence interval, or as percentage frequencies. A Student's t-test was used to assess differences between T-1 and T0 ("washout" period). A repeated measure analysis of variance (ANOVA) was used to evaluate within-subjects' differences for all outcome measures in the different assessment periods and a pairwise analysis was used to compare between the different assessment points. Statistical significance was set at $p<0.05$.

\section{Data availability}

The data associated with the paper are not publicly available but are available from the corresponding author on reasonable request.

\section{Results}

\section{Participants' demographics}

The clinical and demographic characteristics of the participants at baseline are presented in Table 1 . Only 3 patients $(1,8$ and 9$)$ presented moderate levels of pain (NPRS $\leq 5)$. Symptoms duration ranged between two months and two years. Three patients $(3,8$ and 10$)$ demonstrated impaired tactile acuity (i.e. larger TPDT) at baseline in the affected shoulder compared to normative values reported for healthy individuals [i.e. 44.8 (13.1) mm] [28]. 80\% of the subjects presented lower accuracy in the LRJT at baseline compared to normative values [30]. This lower accuracy was observed bilaterally in $50 \%$ of the subjects and in the affected side in $30 \%$. Only 2 patients ( 1 and 8 ) were slower in the LRJT in the affected shoulder compared to normative values [30]. Six patients were slower in the LRJT in the non-dominant shoulder. 
Table 1

\begin{tabular}{|c|c|c|c|c|c|c|c|c|c|c|c|}
\hline \multicolumn{12}{|l|}{ Patient } \\
\hline & & 1 & 2 & 3 & 4 & 5 & 6 & 7 & 8 & 9 & 10 \\
\hline Age (years) & & 51 & 51 & 49 & 49 & 46 & 63 & 59 & 58 & 48 & 47 \\
\hline Sex (male/female) & & $f$ & $f$ & $f$ & $f$ & $f$ & $f$ & $\mathrm{~m}$ & f & $f$ & $\mathrm{~m}$ \\
\hline Weight (kg) & & 53 & 57 & 85 & 55 & 55 & 74 & 60 & 63 & 63 & 75 \\
\hline Length (cm) & & 169 & 164 & 175 & 166 & 155 & 164 & 170 & 162 & 168 & 189 \\
\hline Affected shoulder & & left & right & right & right & right & right & right & left & left & left \\
\hline Dominant Side & & right & right & right & right & right & right & right & right & right & right \\
\hline Symptoms duration (months) & & 2 & 15 & 6 & 6 & 16 & 12 & 3 & 3 & 24 & 10 \\
\hline SPADI (0-100) & & 91.54 & 26.15 & 20 & 59.23 & 20 & 74.62 & 40.77 & 75.38 & 62.31 & 54.62 \\
\hline NPRS $(0-10)$ & & 5 & 2 & 1 & 3 & 3 & 0 & 1 & 5 & 5 & 2 \\
\hline $\begin{array}{l}\text { PER ROM } \\
\text { (degrees) }\end{array}$ & & 6 & 24 & 34 & 0 & 56 & 55 & 14 & 28 & 18 & 43 \\
\hline AF ROM (degrees) & & 60 & 110 & 102 & 66 & 156 & 150 & 86 & 78 & 118 & 140 \\
\hline TPD threshold (mm) & & 22.5 & 35 & 120 & 37.5 & 35 & 20 & 27.5 & 50 & 20 & 57.5 \\
\hline \multirow[t]{2}{*}{ Left/right accuracy (\%) } & Left & 87 & 100 & 100 & 100 & 100 & 73 & 93 & 93 & 100 & 93 \\
\hline & Right & 87 & 93 & 80 & 10 & 80 & 67 & 87 & 73 & 100 & 93 \\
\hline \multirow[t]{2}{*}{ Left/right speed (s) } & Left & 1.8 & 1.9 & 1.8 & 1.8 & 2.2 & 2 & 1.4 & 2.5 & 1.2 & 1.6 \\
\hline & Right & 2 & 1.6 & 1.2 & 1.4 & 1.4 & 1.4 & 1.3 & 1.7 & 1.3 & 1.8 \\
\hline PCS (0-52) & & 11 & 4 & 0 & 2 & 35 & 13 & 23 & 18 & 19 & 18 \\
\hline CSI (0-100) & & 47 & 16 & 29 & 16 & 54 & 36 & 21 & 45 & 15 & 10 \\
\hline TSK-11 (11-44) & & 35 & 16 & 15 & 15 & 32 & 21 & 27 & 20 & 33 & 36 \\
\hline
\end{tabular}

\section{Primary outcomes}

Self-reported shoulder pain and disability for each patient in the different assessment points is presented in Table 2. SPADI scores improved after treatment in the different assessment points $(p=0.001)$. Significant changes in SPADI scores between baseline and follow-up $(T 0-T 6)(p=0.008)$, but not between baseline and post-treatment (T0-T3) or between post-treatment and follow-up (T3-T6) were observed. 
Table 2

\begin{tabular}{|c|c|c|c|c|c|c|c|c|c|c|c|c|c|c|c|c|}
\hline \multirow[t]{2}{*}{ Patient } & \multicolumn{4}{|c|}{ SPADI (0-100) } & \multicolumn{4}{|c|}{ TSK-11 (11-44) } & \multicolumn{4}{|c|}{ CSI (0-100) } & \multicolumn{4}{|c|}{ PCS (0-52) } \\
\hline & $\mathrm{T}-1$ & TO & T3 & T6 & $\mathrm{T}-1$ & T0 & T3 & T6 & $\mathrm{T}-1$ & TO & T3 & T6 & $\mathrm{T}-1$ & T0 & T3 & T6 \\
\hline 1 & 91.54 & 91.54 & 85.38 & 72.31 & 35 & 35 & 37 & 38 & 47 & 47 & 40 & 41 & 11 & 11 & 13 & 22 \\
\hline 2 & 26.15 & 22.31 & 29.23 & 25.38 & 16 & 14 & 15 & 15 & 16 & 18 & 20 & 14 & 4 & 2 & 2 & 5 \\
\hline 3 & 20 & 20 & & & 15 & 15 & & & 29 & 29 & & & 0 & 0 & & \\
\hline 4 & 59.23 & 59.23 & 57.69 & 20 & 15 & 15 & 16 & 14 & 16 & 16 & 22 & 18 & 2 & 2 & 1 & 0 \\
\hline 5 & 20 & 13.08 & & & 32 & 28 & & & 54 & 47 & & & 35 & 21 & & \\
\hline 6 & 74.62 & 64.62 & 57.69 & 30.77 & 21 & 20 & 16 & 19 & 36 & 39 & 39 & 39 & 13 & 5 & 9 & 11 \\
\hline 7 & 40.77 & 40.77 & 4.62 & 2.31 & 27 & 27 & 19 & 18 & 21 & 21 & 17 & 8 & 23 & 23 & 0 & 0 \\
\hline 8 & 75.38 & 76.15 & 6.92 & 0 & 20 & 21 & 11 & 11 & 45 & 46 & 38 & 42 & 18 & 14 & 0 & 0 \\
\hline 9 & 62.31 & 59.23 & 6.15 & 1.54 & 33 & 25 & 17 & 13 & 15 & 15 & 6 & 9 & 19 & 18 & 4 & 1 \\
\hline 10 & 54.62 & 44.62 & 5.38 & 3.08 & 36 & 36 & 28 & 27 & 10 & 10 & 13 & 4 & 18 & 18 & 17 & 11 \\
\hline \multirow{2}{*}{$\begin{array}{l}\text { Mean } \pm \\
\text { SD }\end{array}$} & 47.6 & 52.4 & 31.6 & 19.4 & 23.9 & 23.6 & 19.9 & 19.4 & 28.9 & 28.8 & 24.4 & 21.9 & 14.3 & 11.4 & 5.8 & 6.3 \\
\hline & \pm 25 & $\stackrel{ \pm}{24.9}$ & $\stackrel{ \pm}{31.5}$ & $\stackrel{ \pm}{24.5}$ & $\stackrel{ \pm}{8.3}$ & \pm 8 & \pm 8.5 & \pm 8.9 & $\stackrel{ \pm}{15.7}$ & \pm 14.7 & \pm 13.04 & \pm 16.1 & \pm 10.7 & \pm 8.6 & \pm 6.5 & \pm 7.9 \\
\hline MD & & 4.8 & -16 & $-28,2$ & & -0.3 & -4 & -4.5 & & -0.1 & -4.5 & -7 & & -2.9 & -8.5 & -8 \\
\hline
\end{tabular}

\section{Secondary Outcomes}

Seven participants (70\%) completed the treatment and all the measurements. The three patients $(3,5$ and 8$)$ that didn't complete the treatment attended three, four and six sessions respectively. They dropped-out due to either difficulty for assisting to clinic sessions or lack of support from relatives to comply with home training. No adverse effects were found during or after the intervention. All patients completed the daily treatment diaries consistently.

No significant changes were found after the washout period for all outcome measures except for the TPDT $(p=0.02)$ and SPADI $(p=0.025)$. A significant decrease in shoulder pain was found after treatment $(p=0.028)$, between post-treatment and follow-up $(p=0.028)$ and between baseline and follow-up ( $p=$ 0.004) (Table 3).

Table 3

\begin{tabular}{|c|c|c|c|c|c|c|c|c|c|c|c|c|}
\hline \multirow[t]{2}{*}{ Patient } & \multicolumn{4}{|c|}{ NPRS (0-10) } & \multicolumn{4}{|c|}{ PER ROM (degrees) } & \multicolumn{4}{|c|}{ AF ROM (degrees) } \\
\hline & $\mathrm{T}-1$ & T0 & T3 & T6 & $\mathrm{T}-1$ & T0 & T3 & T6 & $\mathrm{T}-1$ & T0 & T3 & T6 \\
\hline 1 & 5.25 & 6 & 2.5 & 1 & 6 & -10 & 2 & 4 & 60 & 52 & 64 & 68 \\
\hline 2 & 1.75 & 1.75 & 1.5 & 0.25 & 24 & 22 & 26 & 12 & 110 & 114 & 120 & 150 \\
\hline 3 & 0.5 & 0,5 & & & 34 & 36 & & & 102 & 100 & & \\
\hline 4 & 3 & 3 & 1.75 & 0.88 & -2 & 18 & 6 & 24 & 66 & 60 & 70 & 110 \\
\hline 5 & 3 & 2.25 & & & 56 & 68 & & & 156 & 142 & & \\
\hline 6 & 0 & 4,5 & 3.25 & 0.25 & 55 & 75 & 72 & 70 & 150 & 142 & 150 & 160 \\
\hline 7 & 1,25 & 1 & 0 & 0 & 14 & 10 & 46 & 54 & 86 & 88 & 124 & 140 \\
\hline 8 & 4.75 & 4.5 & 1.25 & 0 & 28 & 25 & 35 & 58 & 78 & 110 & 150 & 160 \\
\hline 9 & 4.75 & 4.5 & 0.25 & 0 & 18 & 30 & 25 & 43 & 118 & 110 & 128 & 158 \\
\hline 10 & 1.5 & 1,5 & 1 & 0 & 43 & 50 & 35 & 60 & 140 & 140 & 155 & 160 \\
\hline \multirow[t]{2}{*}{ Mean \pm SD } & 2.6 & 2.9 & 1.4 & 0.3 & 27.6 & 32.4 & 30.9 & 40.6 & 106.6 & 105.8 & 120.1 & 138.3 \\
\hline & \pm 1.9 & \pm 1.8 & \pm 1.1 & \pm 0.4 & \pm 19.6 & \pm 25.9 & \pm 22.3 & \pm 24.4 & \pm 34.4 & \pm 32.1 & \pm 35.3 & \pm 33.1 \\
\hline MD & & 0.3 & -1.2 & -2.3 & & 4.8 & 3.3 & 13 & & -0.8 & 13.5 & 31.7 \\
\hline
\end{tabular}

Significant improvements were found for active shoulder flexion $(p=0.000)$. Additionally, a significant improvement in active shoulder flexion after treatment $(p=0.016)$, between post-treatment and follow-up $(p=0.020)$ and between baseline and follow-up $(p=0.001)$ was found. ROM values for each patient over 
time are presented in Table 3.

There were no significant changes in tactile acuity or laterality judgement performance over time (Table 4). No significant changes were found in TSK-11, PCS or CSI at any measurement point.

Table 4

\begin{tabular}{|c|c|c|c|c|c|c|c|c|c|c|c|c|c|c|c|c|c|c|c|c|}
\hline \multirow[t]{3}{*}{ Patient } & \multirow{2}{*}{\multicolumn{4}{|c|}{ TPD threshold }} & \multicolumn{8}{|c|}{$\begin{array}{l}\text { Laterality judgement } \\
\text { (right shoulder) }\end{array}$} & \multicolumn{8}{|c|}{$\begin{array}{l}\text { Laterality judgement } \\
\text { (left shoulder) }\end{array}$} \\
\hline & & & & & \multicolumn{4}{|c|}{ Accuracy (\%) } & \multicolumn{4}{|c|}{ Speed (s) } & \multicolumn{4}{|c|}{ Accuracy (\%) } & \multicolumn{4}{|c|}{ Speed (s) } \\
\hline & $\mathrm{T}-1$ & T0 & T3 & T6 & $\mathrm{T}-1$ & TO & T3 & T6 & $\mathrm{T}-1$ & T0 & T3 & T6 & $\mathrm{T}-1$ & T0 & T3 & T6 & $\mathrm{T}-1$ & T0 & T3 & $\mathrm{TE}$ \\
\hline 1 & 22.5 & 30 & 15 & 22.5 & 87 & 87 & 87 & 100 & 2 & 1.8 & 1.2 & 1.6 & 87 & 100 & 100 & 100 & 1.8 & 2.4 & 2.3 & 1.1 \\
\hline 2 & 35 & 30 & 50 & 52.5 & 93 & 93 & 100 & 100 & 1.6 & 1.6 & 1.3 & 1.5 & 100 & 100 & 100 & 93 & 1.9 & 2.4 & 1.3 & $1 .:$ \\
\hline 3 & 120 & 105 & & & 80 & 93 & & & 1.2 & 1.3 & & & 100 & 87 & & & 1.8 & 1.4 & & \\
\hline 4 & 37.5 & 27.5 & 27.5 & 30 & 100 & 100 & 100 & 100 & 1.4 & 1.3 & 1.1 & 1.5 & 100 & 87 & 100 & 93 & 1.8 & 1.4 & 1.4 & 1. \\
\hline 5 & 35 & 27.5 & & & 80 & 100 & & & 1.4 & 1.5 & & & 100 & 100 & & & 2.2 & 1.3 & & \\
\hline 6 & 20 & 10 & 20 & 17.5 & 67 & 47 & 87 & 93 & 1.4 & 0.8 & 1.5 & 1.3 & 73 & 93 & 100 & 93 & 2 & 3.2 & 1.8 & 1.1 \\
\hline 7 & 27.5 & 20 & 22.5 & 15 & 87 & 100 & 100 & 93 & 1.3 & 1.6 & 1.4 & 1.3 & 93 & 100 & 100 & 100 & 1.4 & 1.5 & 1.5 & 1. \\
\hline 8 & 50 & 40 & 20 & 25 & 73 & 80 & 93 & 87 & 1.7 & 1.2 & 1.6 & 1.7 & 93 & 93 & 100 & 67 & 2.5 & 1.7 & 2.3 & $1 .:$ \\
\hline 9 & 20 & 25 & 32.5 & 27.5 & 100 & 100 & 100 & 100 & 1.3 & 1.5 & 1.2 & 1 & 100 & 93 & 93 & 100 & 1.2 & 1.2 & 1.1 & 1.. \\
\hline 10 & 57.5 & 42.5 & 37.5 & 30 & 93 & 100 & 100 & 100 & 1.8 & 1.8 & 1 & 1.1 & 93 & 93 & 100 & 100 & 1.6 & 1.4 & 1 & 1. \\
\hline \multirow{2}{*}{$\begin{array}{l}\text { Mean } \\
\pm S D\end{array}$} & 42.5 & 35.8 & 28.1 & 27.5 & 86 & 90 & 95.9 & 96.6 & 1.5 & 1.4 & 1.3 & 1.4 & 93.9 & 94.6 & 99.1 & 93.3 & 1.8 & 1.8 & 1.6 & 1. \\
\hline & $\stackrel{ \pm}{29.9}$ & $\stackrel{ \pm}{26.1}$ & $\stackrel{ \pm}{11.5}$ & $\stackrel{ \pm}{11.5}$ & $\frac{ \pm}{11.03}$ & $\stackrel{ \pm}{16.6}$ & $\stackrel{ \pm}{5.9}$ & $\frac{ \pm}{5.01}$ & $\stackrel{ \pm}{0.3}$ & $\begin{array}{l} \pm \\
0.3\end{array}$ & $\begin{array}{l} \pm \\
0.2\end{array}$ & $\begin{array}{l} \pm \\
0.2\end{array}$ & $\frac{ \pm}{8.7}$ & $\frac{ \pm}{5.2}$ & $\frac{ \pm}{2.5}$ & $\stackrel{ \pm}{11.2}$ & $\stackrel{ \pm}{0.4}$ & $\begin{array}{l} \pm \\
0.7\end{array}$ & $\begin{array}{l} \pm \\
0.5\end{array}$ & \pm \\
\hline MD & & -6.7 & -14.4 & -15 & & 4 & 9.9 & 10.6 & & -0.1 & -0.2 & -0.1 & & 0.7 & 5.2 & -0.6 & & 0 & -0.2 & -0 . \\
\hline
\end{tabular}

TPDT, Two Point Discrimination Threshold; MD, mean difference.

\section{Discussion}

The main goal of this study was to evaluate the feasibility of implementing a CNS-focused treatment program for people with FS. Further, we aimed to assess the clinical impact of this program on pain and function. Overall no significant changes were found after the washout period thus suggesting minimal changes in the participants' clinical condition before treatment. Our findings revealed medium adherence of participants (70\%) to the CNS-focused treatment and follow-up measurements. Regarding clinical impact, improvements in shoulder pain and active shoulder flexion were shown after treatment and at three months follow-up and in disability at three months follow-up. No significant changes were observed in tactile acuity, laterality judgement, pain catastrophization, fear avoidance or central sensitization after treatment or at follow-up.

Average participants' compliance with treatment was lower than expected. Participants' compliance was recorded with a treatment diary which was consistently fulfilled by all participants, but it was not enough to engage all of them to comply with the totality of treatment as previouly reported by Moseley et al. [23]. Nevertheless, all participants who attended the totality of treatment sessions at the clinic also met the home training dosage. In the current study drop-outs were mainly due to lack of time of a relative to assist participants with their home training tasks. Previous studies also emphasized the difficulties with implementing CNS-focused techniques, in particular home training tasks, due to the lack of "helpers" availability or lack of time from participants [17, 37]. These findings highlight the importance of having a cooperative context when using this kind of therapeutic approach at home. Long-term follow-up of participants was almost feasible as eight participants were followed-up. Only two participants were lost to follow-up as they decided to discontinue the clinical sessions due to difficulties in the conciliation of their work schedules or lack of assistance with home training tasks.

Regarding clinical outcomes, positive effects on pain and shoulder function were observed after treatment which agrees with previous studies using a similar protocol [13]. Specifically, improvements in shoulder pain and active shoulder flexion both after treatment and follow-up measurements and disability scores at follow-up were found. Regarding disability, the change in SPADI scores at follow-up exceeded the MDC and MCID established for individuals with FS and non-specific shoulder pain, respectively $[22,38]$. Likewise, changes in pain intensity after treatment and at follow-up and in active shoulder flexion after treatment and at follow-up also surpassed the MCID established for pain intensity (1.1 points) and MDC for active shoulder flexion ( $\left.11^{\circ}\right)$ in people with shoulder pain, respectively [25,27]. No significant changes were found in the LRJT and TPDT neither after treatment nor at follow-up. To our knowledge, responsiveness to treatment of these two variables in people with FS had not been previously investigated except for a single case report by Sawyer et al. [13], where a $10 \mathrm{~mm}$ TPDT reduction and improvement of accuracy and response time in the LRJT task were observed after intervention [13]. Fekos et al. [39] investigated the efficacy of a treatment combining GMI with mirror therapy in a case-series study of five patients with diferent shoulder painful conditions including one patient with FS. After treatment, all patients showed significant improvements in pain intensity, active shoulder flexion and motor imagery ability, but no significant changes on laterality judgement were found [39]. 
No significant changes in fear avoidance or pain catastrophization were found after treatment. This is not surprising given the nature of the CNS-focused treatment program which mainly included sensory discrimination training and GMI. These two interventions are not expected to address fear or pain catastrophization. Pain neuroscience education has been shown to be effective in this regard for reducing psychosocial factors [40], but only a short discussion of pain from a pain neuroscience perspective was implemented in this study. This may explain the lack of change in psychosocial variables.

To our knowledge, a CNS-focused treatment had not been used before specifically for people with FS except in a case report [13]. However, the aforementioned study did not include home training sessions. In contrast, the present study integrated both clinic and home training sessions which was considered essential to really investigate the feasibility of applying this kind of approach in clinical practice.

\section{Study Limitations}

Our results need to be interpreted in light of some limitations. This feasibility study recruited a sample of ten participants with FS. Despite the reported significant improvements in pain, disability and ROM, clinical effects must be interpretated with caution as a greater sample of participants is needed to better estimate the utility of this treatment for people with FS. Another important limitation is the lack of a control group so future research should overcome this issue. Overall, this study identified key feasibility issues related to home training compliance that suggest a reflection when using this approach, specially concerning the need of support from relatives.

\section{Conclusions}

The results of this feasibility study suggest that a CNS-focused treatment program might be a suitable approach to improve pain and disability in people with FS but further research is needed to draw firm conclusions. Although a high percentage of the sample completed the whole treatment program, some engagement issues arised such as the need for the patient to have a cooperative context when implementing this treatment at home.

\section{Declarations}

\section{Acknowledgments}

The authors would like to thank all the participants who took part in the study. We would also like to thank Dr. Jose Enrique Aroca and Dr. Marta Navarro for their assistance with participants recruitment.

\section{Authors' contributions}

SM applied the treatment to patients and took the lead in writing the manuscript receiving input from all authors, who provided critical feedback and helped shape the research, statistical analysis and manuscript. ALS and SNL contributed to the study design and methodology selected for the assessments and MB, LD and QLL performed recruitment of the sample and performed the patients' assessments. SM performed the statystycal analysis under supervision of QLL, AL, ALS and SNL who assessed in the interpretation of the results. All authors discussed the results and contributed to the final version of the manuscript. AL checked the spelling and grammar of the manuscript. All authors read and approved the final version of the manuscript.

\section{Funding}

This research did not receive any specific grant from funding agencies in the public, commercial, or not-for-profit sectors.

\section{Availability of data and materials}

The manual and de-identified datasets analysed during the current study are available from the corresponding author on reasonable request.

\section{Ethics approval and consent to participate}

The study was approved by the Ethical Committee of the University of Valencia in June 2017 (reference number H1532330957968) and all procedures were performed in accordance with the Declaration of Helsinki.

\section{Consent for publication}

All participants gave their written informed consent prior to participate in the study. The manuscript does not contain data from any individual person that is identifiable.

\section{Competing interests}

The authors affirm that they have no affiliations with or financial involvement in any organization or entity with a direct financial interest in any matter included in this manuscript.

\section{References}

1. Ryan V, Brown H, Lowe CJM, Lewis JS. The pathophysiology associated with primary (idiopathic) frozen shoulder: A systematic review. BMC Musculoskelet Disord. 2016;17:340.

2. Durall CJ. Adhesive Capsulitis (Frozen Shoulder). Clin Orthop Rehabil Team Approach E-Book. 2017;158. 
3. Fields BK, Skalski MR, Patel DB, White EA, Tomasian A, Gross JS, et al. Adhesive capsulitis: review of imaging findings, pathophysiology, clinical presentation, and treatment options. Skeletal Radiol. 2019;1-14.

4. Noten S, Meeus M, Stassijns G, Van Glabbeek F, Verborgt O, Struyf F. Efficacy of different types of mobilization techniques in patients with primary adhesive capsulitis of the shoulder: a systematic review. Arch Phys Med Rehabil. 2016;97:815-825.

5. Gallacher S, Beazley JC, Evans J, Anaspure R, Silver D, Redfern A, et al. A randomized controlled trial of arthroscopic capsular release versus hydrodilatation in the treatment of primary frozen shoulder. J Shoulder Elbow Surg. 2018;27:1401-1406.

6. Struyf F, Meeus M. Current evidence on physical therapy in patients with adhesive capsulitis: what are we missing? Clin Rheumatol. 2014;33:593-600.

7. Sanchis MN, Lluch E, Nijs J, Struyf F, Kangasperko M. The role of central sensitization in shoulder pain: a systematic literature review. Semin Arthritis Rheum [Internet]. Elsevier; 2015. p. 710-716. Available from: http://www.sciencedirect.com/science/article/pii/S0049017214002765

8. Noten S, Struyf F, Lluch E, D'hoore M, Van Looveren E, Meeus M. Central pain processing in patients with shoulder pain: a review of the literature. Pain Pract. 2017;17:267-280.

9. Haik MN, Evans K, Smith A, Henríquez L, Bisset L. People with musculoskeletal shoulder pain demonstrate no signs of altered pain processing. Musculoskelet Sci Pract. 2019;39:32-38.

10. Bowering KJ, O'Connell NE, Tabor A, Catley MJ, Leake HB, Moseley GL, et al. The effects of graded motor imagery and its components on chronic pain: a systematic review and meta-analysis. J Pain. 2013;14:3-13.

11. Kälin S, Rausch-Osthoff A-K, Bauer CM. What is the effect of sensory discrimination training on chronic low back pain? A systematic review. BMC Musculoskelet Disord. 2016;17:143.

12. Louw A, Puentedura EJ, Reese D, Parker P, Miller T, Mintken PE. Immediate effects of mirror therapy in patients with shoulder pain and decreased range of motion. Arch Phys Med Rehabil. 2017;98:1941-1947.

13. Sawyer EE, McDevitt AW, Louw A, Puentedura EJ, Mintken PE. Use of Pain Neuroscience Education, Tactile Discrimination, and Graded Motor Imagery in an Individual With Frozen Shoulder. J Orthop Sports Phys Ther. 2018;48:174-184.

14. Lewis J. Frozen shoulder contracture syndrome-Aetiology, diagnosis and management. Man Ther. 2015;20:2-9.

15. Kelley MJ, Mcclure PW, Leggin BG. Frozen shoulder: evidence and a proposed model guiding rehabilitation. J Orthop Sports Phys Ther. 2009;39:135-148.

16. Zuckerman JD, Rokito A. Frozen shoulder: a consensus definition. J Shoulder Elbow Surg. 2011;20:322-325.

17. Harms A, Heredia-Rizo AM, Moseley GL, Hau R, Stanton TR. A feasibility study of brain-targeted treatment for people with painful knee osteoarthritis in tertiary care. Physiother Theory Pract. 2020;36:142-56.

18. Louw A, Diener I, Butler DS, Puentedura EJ. The effect of neuroscience education on pain, disability, anxiety, and stress in chronic musculoskeletal pain. Arch Phys Med Rehabil. 2011;92:2041-2056.

19. Schmidt S, Ferrer M, González M, González N, Valderas JM, Alonso J, et al. Evaluation of shoulder-specific patient-reported outcome measures: a systematic and standardized comparison of available evidence. J Shoulder Elbow Surg. 2014;23:434-444.

20. Roach KE, Budiman-Mak E, Songsiridej N, Lertratanakul Y. Development of a shoulder pain and disability index. Arthritis Rheum Off J Am Coll Rheumatol. 1991;4:143-149.

21. Membrilla-Mesa MD, Cuesta-Vargas Al, Pozuelo-Calvo R, Tejero-Fernández V, Martín-Martín L, Arroyo-Morales M. Shoulder pain and disability index: cross cultural validation and evaluation of psychometric properties of the Spanish version. Health Qual Life Outcomes. 2015;13:200.

22. Roy J-S, MacDermid JC, Woodhouse LJ. Measuring shoulder function: a systematic review of four questionnaires. Arthritis Care Res Off $\mathrm{J}$ Am Coll Rheumatol. 2009;61:623-632.

23. Moseley GL. Do training diaries affect and reflect adherence to home programs? Arthritis Care Res Off J Am Coll Rheumatol. 2006;55:662-664.

24. Kroenke K, Bair MJ, Damush TM, Wu J, Hoke S, Sutherland J, et al. Optimized antidepressant therapy and pain self-management in primary care patients with depression and musculoskeletal pain: a randomized controlled trial. Jama. 2009;301:2099-2110.

25. Mintken PE, Glynn P, Cleland JA. Psychometric properties of the shortened disabilities of the Arm, Shoulder, and Hand Questionnaire (QuickDASH) and Numeric Pain Rating Scale in patients with shoulder pain. J Shoulder Elbow Surg. 2009;18:920-926.

26. Kolber MJ, Hanney WJ. The reliability and concurrent validity of shoulder mobility measurements using a digital inclinometer and goniometer: a technical report. Int J Sports Phys Ther. 2012;7:306.

27. Muir SW, Corea CL, Beaupre L. Evaluating change in clinical status: reliability and measures of agreement for the assessment of glenohumeral range of motion. North Am J Sports Phys Ther NAJSPT. 2010;5:98.

28. Botnmark I, Tumilty S, Mani R. Tactile acuity, body schema integrity and physical performance of the shoulder: a cross-sectional study. Man Ther. 2016;23:9-16.

29. Wallwork SB, Butler DS, Fulton I, Stewart H, Darmawan I, Moseley GL. Left/right neck rotation judgments are affected by age, gender, handedness and image rotation. Man Ther. 2013;18:225-230.

30. Breckenridge JD, McAuley JH, Butler DS, Stewart H, Moseley GL, Ginn KA. The development of a shoulder specific left/right judgement task: validity \& reliability. Musculoskelet Sci Pract. 2017;28:39-45.

31. Gómez-Pérez L, López-Martínez AE, Ruiz-Párraga GT. Psychometric properties of the Spanish version of the Tampa Scale for Kinesiophobia (TSK). J Pain. 2011;12:425-435.

32. Kori SH. Kinisophobia: a new view of chronic pain behavior. Pain Manage. 1990;35-43. 
33. Hapidou EG, O'Brien MA, Pierrynowski MR, de las Heras E, Patel M, Patla T. Fear and avoidance of movement in people with chronic pain: psychometric properties of the 11-Item Tampa Scale for Kinesiophobia (TSK-11). Physiother Can. 2012;64:235-241.

34. Cuesta-Vargas Al, Roldan-Jimenez C, Neblett R, J. Gatchel R. Cross-cultural adaptation and validity of the Spanish central sensitization inventory. Springer Plus. 2016;5:1837.

35. Sullivan MJ, Bishop SR, Pivik J. The pain catastrophizing scale: development and validation. Psychol Assess. 1995;7:524.

36. Lluch-Girbés E, Dueñas L, Mena-del Horno S, Luque-Suarez A, Navarro-Ledesma S, Louw A. A central nervous system-focused treatment approach for people with frozen shoulder: protocol for a randomized clinical trial. Trials. 2019;20:498.

37. Johnson S, Hall J, Barnett S al, Draper M, Derbyshire G, Haynes L, et al. Using graded motor imagery for complex regional pain syndrome in clinical practice: failure to improve pain. Eur J Pain. 2012;16:550-561.

38. Tveitlaa EK, Ekeberg OM, Juel NG, Bautz-Holter E. Responsiveness of the shoulder pain and disability index in patients with adhesive capsulitis. BMC Musculoskelet Disord. 2008;9:161.

39. Fekos C, Kallistratou A, Fousekis K, lakovidis P, Kottaras S. Modified Graded Motor Imagery Programme Containing "Fekos Mirror Therapy Method”: A Novel Therapeutic Method for the Treatment of Shoulder Dysfunctions-A Pilot Study. J Nov Physiother. 2017;7:2.

40. Louw A, Zimney K, Puentedura EJ, Diener I. The efficacy of pain neuroscience education on musculoskeletal pain: a systematic review of the literature. Physiother Theory Pract. 2016;32:332-355.

\section{Figures}

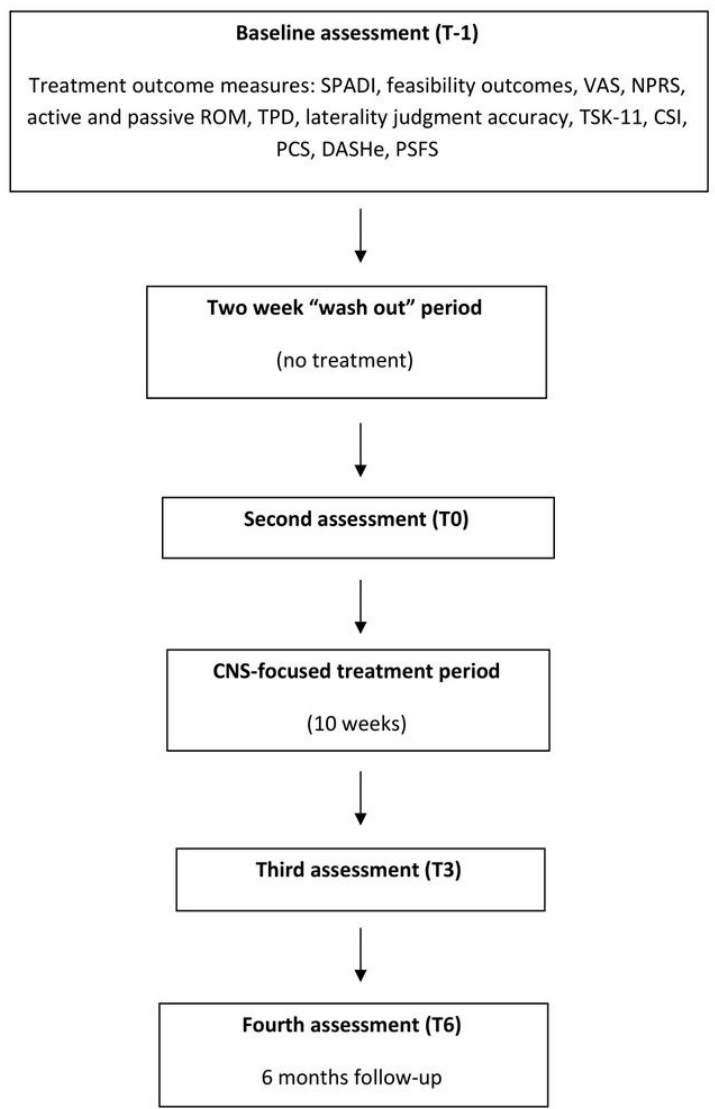

Figure 1

Figure 1

\section{Supplementary Files}

This is a list of supplementary files associated with this preprint. Click to download. 
- TIDieRChecklistWord.docx

Page $11 / 11$ 